\title{
New Exact Solutions for the Coupled Nonlinear Schrödinger Equations with Variable Coefficients
}

\author{
Yuting Qiu*, Ping Gao \\ School of Mathematics and Information Science, Guangzhou University, Guangzhou, China \\ Email: *victoriayutingqiu@163.com, 1554744857@qq.com
}

How to cite this paper: Qiu, Y.T. and Gao, P. (2020) New Exact Solutions for the Coupled Nonlinear Schrödinger Equations with Variable Coefficients. Journal of Applied Mathematics and Physics, 8, 1515-1523. https://doi.org/10.4236/jamp.2020.88117

Received: July 20, 2020

Accepted: August 15, 2020

Published: August 18, 2020

Copyright (๑) 2020 by author(s) and Scientific Research Publishing Inc. This work is licensed under the Creative Commons Attribution International License (CC BY 4.0).

http://creativecommons.org/licenses/by/4.0/

\begin{abstract}
In this paper, coupled nonlinear Schrödinger equations with variable coefficients are studied, which can be used to describe the interaction among the modes in nonlinear optics and Bose-Einstein condensation. Some novel bright-dark solitons and dark-dark solitons are obtained by modified SineGordon equation method. Moreover, some figures are provided to illustrate how the soliton solutions propagation is determined by the different values of the variable group velocity dispersion terms, which can be used to model various phenomena.
\end{abstract}

\section{Keywords}

Modified Sine-Gordon Equation Method, Coupled Nonlinear Schrödinger Equation, Exact Solutions, Bright-Dark Soliton

\section{Introduction}

In nonlinear optics, the coupled nonlinear Schrödinger (CNLS) equations are often used to describe propagation of optical soliton in birefringence fibers, multimode fibers and optical fiber arrays. Many researchers have studied the CNLS equation with constant coefficient. In recent years, a number of methods are used to solve the coupled integrable nonlinear models, such as Hirota bilinear method [1] [2] [3], Painlev analysis method [4], Function expansion method [5] and direct perturbation method [6] and so on. However, the evolutions of vector solitons for CNLS equation with constant coefficients are not dependent on any controllable parameters. With the development of modern science, people need to manage and control soliton propagation, which will make solitons into practical information carriers. Therefore, variable coefficient equation has more prac- 
tical significance.

In this paper, we will consider the following coupled nonlinear Schrödinger equation with variable coefficients (VCNLS) [7]:

$$
\begin{aligned}
& i \psi_{1 t}+a(t) \psi_{1 x x}+\left(b_{1}(t)\left|\psi_{1}\right|^{2}+b_{2}(t)\left|\psi_{2}\right|^{2}\right) \psi_{1}+v(t) \psi_{1}=0, \\
& i \psi_{2 t}+a(t) \psi_{2 x x}+\left(b_{1}(t)\left|\psi_{1}\right|^{2}+b_{2}(t)\left|\psi_{2}\right|^{2}\right) \psi_{2}+v(t) \psi_{2}=0 .
\end{aligned}
$$

where $\psi_{1}$ and $\psi_{2}$ are complex envelopes of the propagating beam of the two modes, and $x, t$ are the spatial coordinate and retarded time respectively. The coefficients $a(t)$ represent the group velocity dispersion; $b_{1}(t), b_{2}(t)$ mean nonlinear interactions and $v(t)$ means external potential. Finding exact solutions of VCNLS, especially soliton solutions [8] [9] have been a subject of great interest to mathematicians and physicists. Han [7] constructed an explicit transformation, which maps VCNLS to the classical CNLS, and obtains Bright-Dark solitons for VCNLS. Exact traveling wave and soliton solutions of the VCNLS equation have been obtained by Zhong [10] using homogeneous balance principle and the F-expansion technique. Yu and Yang [11] presented the similarity transformations for this system. Because of the complexity of VCNLS form, it is difficult to solve it directly, so the study of this kind of soliton solutions is not so extensive.

The paper is organized as follows. Section 2 describes the modified Sine-Gordon equation method [12]. In Section 3, we have applied the method to the VCNLS, and derive some bright-dark solitons and dark-dark solitons. Section 4 is devoted to analysis of shape changing exhibited by these soliton solutions when variable coefficients are altered. In the last section, Section 5, conclusion is presented.

\section{The Method}

Let us consider a form of a nonlinear partial differential equation

$$
H_{k}\left(x, t, \frac{\partial \psi_{k}}{\partial x}, \frac{\partial \psi_{k}}{\partial t}, \frac{\partial^{2} \psi_{k}}{\partial x^{2}}, \cdots, a(t), b_{1}(t), b_{2}(t), v(t)\right)=0,
$$

where $a(t), b_{k}(t)$ and $v(t)$ are arbitrary functions in $t$ and $k=1,2$. In the following, we offer the main steps of this method:

Step 1: Use the following assumptions:

$$
\begin{aligned}
& \psi_{1}(x, t)=U_{1}(\zeta) \mathrm{e}^{i(\alpha x-\theta(t))}, \\
& \psi_{2}(x, t)=U_{2}(\zeta) \mathrm{e}^{i(\alpha x-\theta(t))} .
\end{aligned}
$$

where $U_{1}(\zeta)$ and $U_{2}(\zeta)$ are the new dependant functions, $\zeta=\mu(x-\lambda(t))$ is the new independent variable, $\lambda(t)$ is an arbitrary function of $t$ and $\mu$ and $\alpha$ are the frequency and the width of the soliton respectively.

Step 2: Collect the coeffients of $U_{1}(\zeta)$ and $U_{2}(\zeta)$ and their derivatives, and then assume the imaginary part is equal to zero.

Step 3: Take the coefficient of the largest linear term as the normalization 
coefficient.

Step 4: The derivatives and powers of $U_{1}(\zeta)$ and $U_{2}(\zeta)$ are equal to the term multiplied by a constant, so the arbitrary functions will be determined, and the Equation (2) is transformed into the following nonlinear ordinary differential system.

$$
Q_{i}\left(U_{i}(\zeta), U_{i}^{\prime}(\zeta), U_{i}^{\prime \prime}(\zeta), \cdots\right)=0
$$

Step 5: Use the solutions of the Sine-Gordon equation [13] [14] by assuming that

$$
\begin{aligned}
& U_{1}(\xi)=\sum_{i=1}^{n} \cos ^{i-1}(w(\xi)) \times\left[B_{i} \sin (w(\xi))+A_{i} \cos (w(\xi))\right]+A_{0}, \\
& U_{2}(\xi)=\sum_{j=1}^{m} \cos ^{j-1}(w(\xi)) \times\left[E_{j} \sin (w(\xi))+D_{j} \cos (w(\xi))\right]+D_{0} .
\end{aligned}
$$

where $A_{i}, B_{i}, D_{i}, E_{i}, A_{0}$ and $D_{0}$ are arbitrary constants and $n$ and $m$ are determined by balancing the most dispersive term and the greatest nonlinear term in Equation (4), and

$$
w^{\prime}(\xi)=\sin (w(\xi))
$$

and

$$
\sin (w(\xi))=\operatorname{sech}(\xi) \text { or } \cos (w(\xi))=\tanh (\xi)
$$

Step 6: Equating the coefficients of $\sin ^{i}(w(\xi))$ and $\cos ^{i}(w(\xi))$ to zero and an algebraic system for the constant $A_{i}, B_{i}, D_{i}, E_{i}, A_{0}$ and $D_{0}$ are obtained, by solving them with a Maple program and back-substituting into Equation (5) and Equation (3) via Equation (7), novel soliton solutions are obtained for the system of Equation (2).

Advantages of the method: The Sine-Gordon equation method has limitations and is suitable for some constant coefficient systems, but modified SineGordon equation method is applicable to systems with variable coefficients containing imaginary parts. As a result, some spanking new solutions might be originated via this method and this method can use computational software like Maple or Mathematica to reduce the amount of computation.

\section{Exact Solutions for VCNLS}

By substituting the assumptions in Equation (3) into Equation (1), we obtain

$$
\begin{aligned}
& a(t) \mu^{2} U_{1}^{\prime \prime}(\xi)+i \mu\left(2 \alpha a(t)-\lambda^{\prime}\right) U_{1}^{\prime}(\xi)+\left(\theta^{\prime}-\alpha^{2} a(t)\right) U_{1}(\xi) \\
& +\left(b_{1}(t) U_{1}^{2}(\xi)+b_{2}(t) U_{2}^{2}(\xi)\right) U_{1}(\xi)+v(t) U_{1}(\xi)=0, \\
& a(t) \mu^{2} U_{2}^{\prime \prime}(\xi)+i \mu\left(2 \alpha a(t)-\lambda^{\prime}\right) U_{2}^{\prime}(\xi)+\left(\theta^{\prime}-\alpha^{2} a(t)\right) U_{2}(\xi) \\
& +\left(b_{1}(t) U_{1}^{2}(\xi)+b_{2}(t) U_{2}^{2}(\xi)\right) U_{2}(\xi)+v(t) U_{2}(\xi)=0 .
\end{aligned}
$$

to make Equation (8) real, the terms $U_{1}^{\prime}(\xi)$ and $U_{2}^{\prime}(\xi)$ must be eliminated, so according to Step 2, we get $\lambda(t)=2 \alpha \int a(t) \mathrm{d} t+\lambda_{0}$, and then follow Step 3, that is, take the coefficients of $U_{1}^{\prime \prime}(\xi)$ and $U_{2}^{\prime \prime}(\xi)$ as the normalized coeffi- 
cients, and we get

$$
\begin{gathered}
\theta(t)=\int\left(\mu^{2} c_{1}+\alpha^{2}\right) a(t) \mathrm{d} t+\theta_{0} \\
\lambda(t)=2 \alpha \int a(t) \mathrm{d} t+\lambda_{0} \\
b_{1}(t)=c_{2} \mu^{2} a(t) \\
b_{2}(t)=c_{3} \mu^{2} a(t) \\
v(t)=c_{4} \mu^{2} a(t)
\end{gathered}
$$

where $c_{1}, c_{2}, c_{3}$ and $c_{4}$ are constants and $\lambda_{0}$ and $\theta_{0}$ is an integration constant. Therefore,

$$
\xi=\mu\left(x-2 \alpha \int a(t) \mathrm{d} t\right)
$$

And, Equation (8) can be simplified as follows

$$
\begin{aligned}
& U_{1}^{\prime \prime}(\xi)+c_{1} U_{1}(\xi)+\left(c_{2} U_{1}^{2}(\xi)+c_{3} U_{2}^{2}(\xi)\right) U_{1}(\xi)+c_{4} U_{1}(\xi)=0, \\
& U_{2}^{\prime \prime}(\xi)+c_{1} U_{2}(\xi)+\left(c_{2} U_{1}^{2}(\xi)+c_{3} U_{2}^{2}(\xi)\right) U_{2}(\xi)+c_{4} U_{2}(\xi)=0 .
\end{aligned}
$$

By balancing the dispersive and nonlinear terms in Equation (15) we get $m+2=2 m+m$ and $n+2=2 n+n$, i.e. $m=n=1$, so according to Step 5, we assume that

$$
\begin{aligned}
& U_{1}(\xi)=B_{1} \sin (w(\xi))+A_{1} \cos (w(\xi))+A_{0}, \\
& U_{2}(\xi)=E_{1} \sin (w(\xi))+D_{1} \cos (w(\xi))+D_{0} .
\end{aligned}
$$

Substituting Equation (16) and the necessary derivatives into Equation (15) using Equation (6), applying trigonometric identities and collecting the coefficients of $\sin (w(\xi))$ and $\cos (w(\xi))$ that are containing independent combinations to zero, and we obtain the following independent parametric equations:

$$
\begin{aligned}
& \sin (w(\xi)): \\
& \quad c_{1} B_{1}+c_{4} B_{1}+3 c_{2} B_{1} A_{0}^{2}+c_{3} B_{1} D_{0}^{2}+2 c_{3} E_{1} D_{0} A_{0}=0 \\
& \cos (w(\xi)): \\
& c_{1} A_{1}+c_{4} A_{1}+3 c_{2} A_{1} A_{0}^{2}+c_{3} A_{1} D_{0}^{2}+2 c_{3} D_{1} D_{0} A_{0}+c_{2} A_{1}^{3}+c_{3} A_{1} D_{1}^{2}=0 \\
& \sin ^{3}(w(\xi)): \\
& \sin (w(\xi)) \cos ^{2}(w(\xi)): \quad-B_{1}+c_{2} B_{1}^{3}+c_{3} B_{1} E_{1}^{2}=0 \\
& B_{1}+3 c_{2} B_{1} A_{1}^{2}+c_{3} B_{1} D_{1}^{2}+2 c_{3} A_{1} E_{1} D_{1}=0 \\
& \sin ^{2}(w(\xi)) \cos (w(\xi)): \\
& -2 A_{1}+3 c_{2} A_{1} B_{1}^{2}+c_{3} A_{1} E_{1}^{2}+2 c_{3} E_{1} D_{1} B_{1}-c_{2} A_{1}^{3}-c_{3} A_{1} D_{1}^{2}=0 \\
& \sin ^{2}(w(\xi)): \\
& \quad 3 c_{2} A_{0} B_{1}^{2}+c_{3} A_{0} E_{1}^{2}+2 c_{3} E_{1} D_{0} B_{1}=0 \\
& \sin ^{2}(w(\xi)) \cos (w(\xi)):
\end{aligned}
$$




$$
6 c_{2} B_{1} A_{1} A_{0}+2 c_{3} D_{1} D_{0} B_{1}+2 c_{3} A_{1} E_{1} D_{0}+2 c_{3} A_{0} E_{1} D_{1}=0
$$

$\cos ^{2}(w(\xi)):$

$$
3 c_{2} A_{0} A_{1}^{2}+2 c_{3} A_{1} D_{1} D_{0}+c_{3} A_{0} D_{1}^{2}=0
$$

constants:

$$
c_{1} A_{0}+c_{4} A_{0}+c_{2} A_{0}^{3}+c_{3} A_{0} D_{0}^{2}=0
$$

Solving Equations (17)-(25), we obtain the following cases and solutions using Equation (7).

Case 1: When $A_{0}=B_{1}=0, c_{2}=-\frac{2}{A_{1}^{2}}, c_{3}=0, c_{4}=-c_{1}+2$, we get the following bright-dark solitons:

$$
\begin{aligned}
\psi_{1}(x, t)= & A_{1} \tanh \left(\mu\left(x-2 \alpha \int a(t) \mathrm{d} t\right)\right) \exp \left(i\left(\alpha x-\int\left(\mu^{2} c_{1}+\alpha^{2}\right) a(t) \mathrm{d} t+\theta_{0}\right)\right), \\
\psi_{2}(x, t)= & {\left[E_{1} \operatorname{sech}\left(\mu\left(x-2 \alpha \int a(t) \mathrm{d} t\right)\right)+D_{1} \tanh \left(\mu\left(x-2 \alpha \int a(t) \mathrm{d} t\right)\right)+D_{0}\right] } \\
& \times \exp \left(i\left(\alpha x-\int\left(\mu^{2} c_{1}+\alpha^{2}\right) a(t) \mathrm{d} t+\theta_{0}\right)\right) .
\end{aligned}
$$

where $A_{1}, D_{1}, E_{1}$ and $c_{1}$ are arbitrary constants.

Case 2: When $A_{0}=B_{1}=D_{0}=E_{1}=0, c_{3}=-\frac{2}{D_{1}^{2}}, c_{4}=-c_{1}$, we get the following dark-dark solitons:

$$
\begin{aligned}
& \psi_{1}(x, t)=A_{1} \tanh \left(\mu\left(x-2 \alpha \int a(t) \mathrm{d} t\right)\right) \exp \left(i\left(\alpha x-\int\left(\mu^{2} c_{1}+\alpha^{2}\right) a(t) \mathrm{d} t+\theta_{0}\right)\right), \\
& \psi_{2}(x, t)=D_{1} \tanh \left(\mu\left(x-2 \alpha \int a(t) \mathrm{d} t\right)\right) \exp \left(i\left(\alpha x-\int\left(\mu^{2} c_{1}+\alpha^{2}\right) a(t) \mathrm{d} t+\theta_{0}\right)\right) .
\end{aligned}
$$

where $A_{1}, D_{1}, C_{1}$ and $C_{2}$ are arbitrary constants.

Case 3: When $A_{0}=A_{1}=D_{0}=E_{1}=0, c_{2}=-\frac{2}{B_{1}^{2}}, c_{3}=-\frac{2}{D_{1}^{2}}, c_{4}=-c_{1}$, we get the following bright-dark solitons:

$$
\begin{aligned}
& \psi_{1}(x, t)=B_{1} \operatorname{sech}\left(\mu\left(x-2 \alpha \int a(t) \mathrm{d} t\right)\right) \exp \left(i\left(\alpha x-\int\left(\mu^{2} c_{1}+\alpha^{2}\right) a(t) \mathrm{d} t+\theta_{0}\right)\right), \\
& \psi_{2}(x, t)=D_{1} \tanh \left(\mu\left(x-2 \alpha \int a(t) \mathrm{d} t\right)\right) \exp \left(i\left(\alpha x-\int\left(\mu^{2} c_{1}+\alpha^{2}\right) a(t) \mathrm{d} t+\theta_{0}\right)\right) .
\end{aligned}
$$

where $B_{1}, D_{1}$, and $c_{1}$ are arbitrary constants.

Case 4: When $A_{0}=B_{1}=D_{0}=D_{1}=0, c_{2}=-\frac{c_{4}+c_{1}}{A_{1}^{2}}, c_{3}=-\frac{c_{4}+c_{1}-2}{E_{1}^{2}}$, we get the following dark-bright solitons:

$$
\begin{aligned}
\psi_{1}(x, t)= & A_{1} \tanh \left(\mu\left(x-2 \alpha \int a(t) \mathrm{d} t\right)\right) \exp \left(i\left(\alpha x-\int\left(\mu^{2} c_{1}+\alpha^{2}\right) a(t) \mathrm{d} t+\theta_{0}\right)\right), \\
\psi_{2}(x, t)= & {\left[E_{1} \operatorname{sech}\left(\mu\left(x-2 \alpha \int a(t) \mathrm{d} t\right)\right)+D_{0}\right] } \\
& \times \exp \left(i\left(\alpha x-\int\left(\mu^{2} c_{1}+\alpha^{2}\right) a(t) \mathrm{d} t+\theta_{0}\right)\right) .
\end{aligned}
$$

where $A_{1}, E_{1}, C_{1}$ and $c_{4}$ are arbitrary constants. 


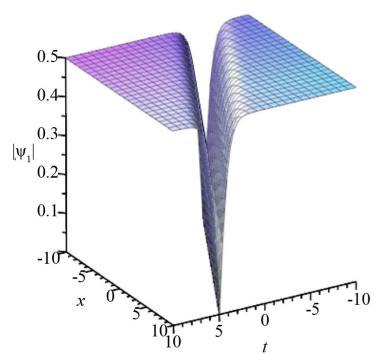

(a)

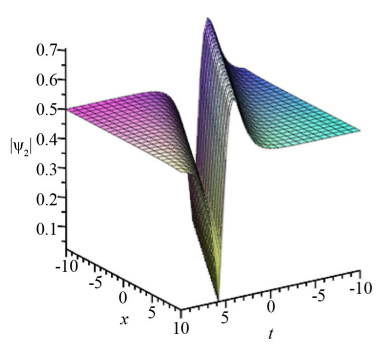

(e)

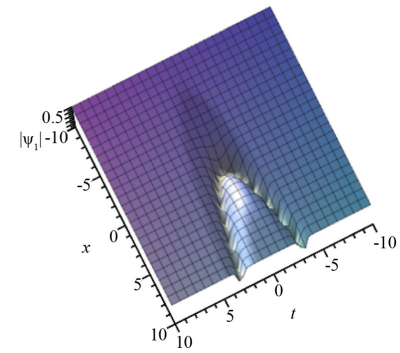

(b)

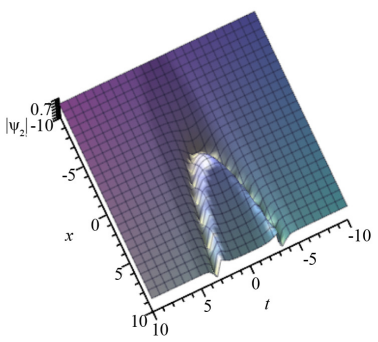

(f)

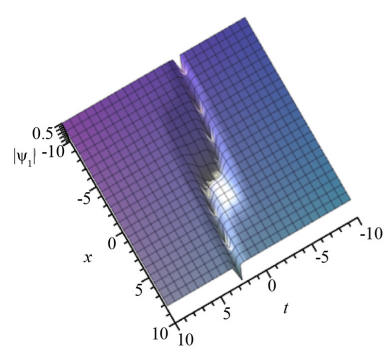

(c)

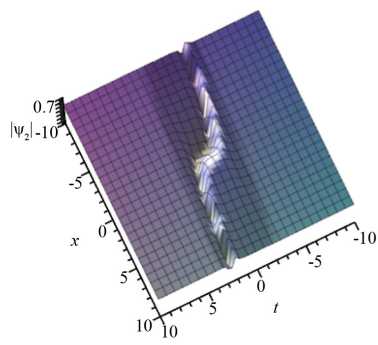

(g)

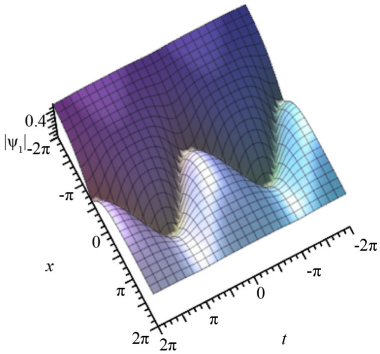

(d)

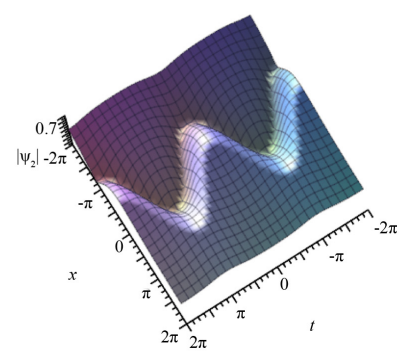

(h)

Figure 1. Evolution of dark-bright soliton solutions of Equation (26), plotted for different values of $a(t)$. (a) $a(t)=1$; (b) $a(t)=t ;(\mathrm{c}) a(t)=t^{2} ;$ (d) $a(t)=\sin (t) ;(\mathrm{e}) a(t)=1 ;(\mathrm{f}) a(t)=t ;(\mathrm{g}) a(t)=t^{2} ;$ (h) $a(t)=\sin (t)$. 


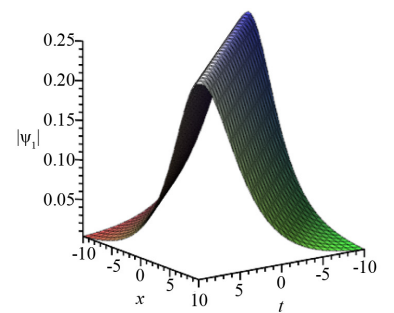

(a)

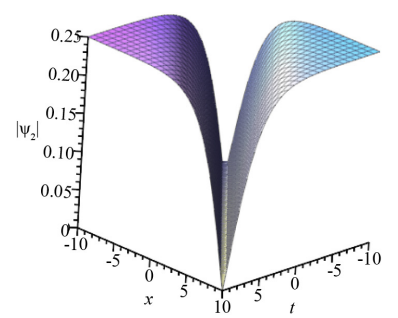

(e)

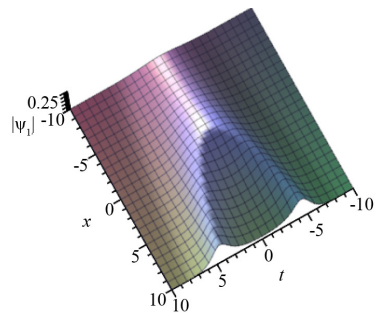

(b)

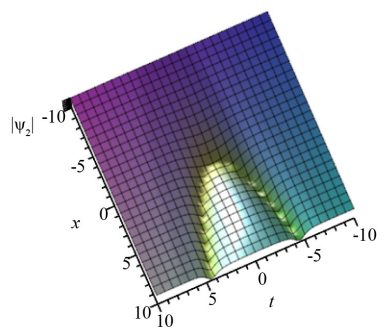

(f)

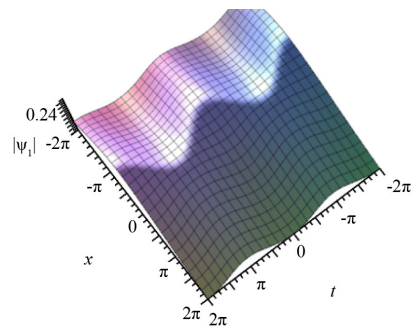

(c)

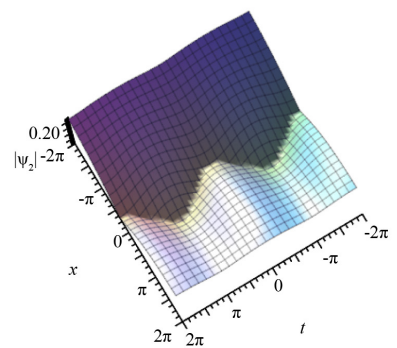

(g)

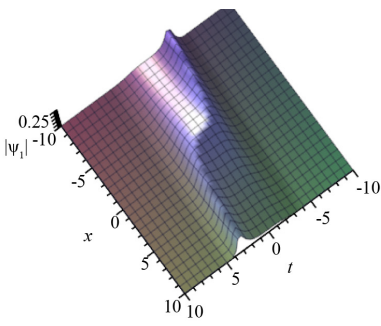

(d)

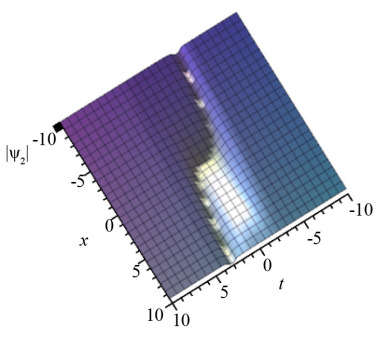

(h)

Figure 2. Evolution of bright-dark soliton solution of Equation (28), plotted for different values of $a(t)$. (a) $a(t)=1$; (b) $a(t)=t ;(\mathrm{c}) a(t)=t^{2} ;$ (d) $a(t)=\sin (t) ;(\mathrm{e}) a(t)=1 ;(\mathrm{f}) a(t)=t ;(\mathrm{g}) a(t)=t^{2} ;$ (h) $a(t)=\sin (t)$.

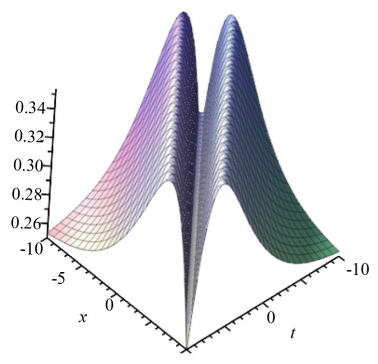

(a)

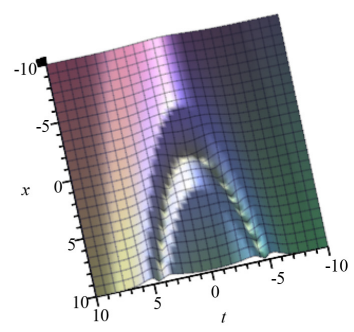

(b)

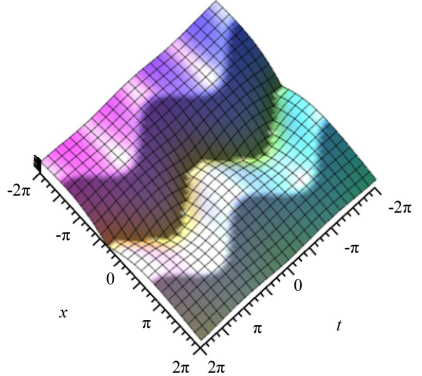

(c)

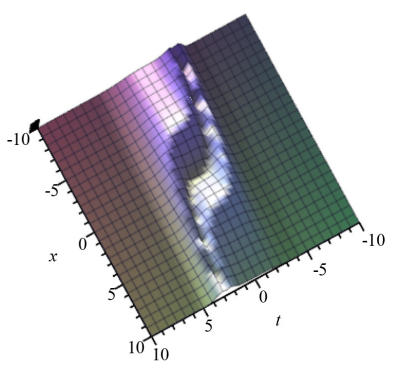

(d)

Figure 3. Evolution and interaction of Equation (28) with the same parameters as Figure 2. (a) $a(t)=1 ;(\mathrm{b}) a(t)=t$; (c) $a(t)=t^{2} ;(\mathrm{d}) \quad a(t)=\sin (t)$.

each soliton shape keeps invariant after interaction, which denotes that the interaction is elasticwe. We can see that the solitons show a periodic property but the solitons are not symmetrical in the $t$ direction, and have the bell-shaped, parabolic, cubic or periodical-oscillating shapes.

\section{Conclusion}

In this paper, we have obtained some bright-dark soliton solutions and dark-dark soliton solutions of the coupled nonlinear Schrödinger equation with variable coefficients, using modified Sine-Gordon equation method. Then, we have discussed the effects of $a(t)$, which is the group velocity dispersion. For bright-dark soliton solutions 26 and 28 , we have chosen $a(t)$ as the constant, linear, quadratic and trigonometric functions, respectively, and the bell-shaped, parabolic, 
cubic and quasi-parabolic solitons have been obtained correspondingly, as shown in Figure 1 and Figure 2, and found that the propagation of the soliton solutions is determined by this value. We also investigated the evolution and interaction between the two solutions, and obtained that each solution shape keeps invariant after interaction and a periodic property in the $t$ direction, as presented in Figure 3. The results show that modified sine-Gordon method gives soliton solutions for variable coefficients systems directly, without difficult calculations and also could be applied to many coupled nonlinear models.

\section{Conflicts of Interest}

The authors declare no conflicts of interest regarding the publication of this paper.

\section{References}

[1] Lixin, M.A. (2014) The Multi-Soliton Solutions to the KdV Equation by Hirota Method. Progress in Applied Mathematics, 8, 30-35.

[2] Jia, T., Chai, Y. and Hao, H. (2016) Multisoliton Solutions and Breathers for the Coupled Nonlinear Schrdinger Equations via the Hirota Method. Mathematical Problems in Engineering, 2016, 1-11. https://doi.org/10.1155/2016/1741245

[3] Gao, Z., Song, S., Zhang, K. and Guo, X. (2017) The Application of Generalized Coupled Higher-Order Nonlinear Schrdinger Equations with Variable Coefficients in Optical Fibers. Optik, 147, 306-320. https://doi.org/10.1016/j.ijleo.2017.08.092

[4] Xie, F. and Chen, Y. (2003) An Algorithmic Method in Painleve Analysis of PDE. Computer Physics Communications, 154, 197-204.

https://doi.org/10.1016/S0010-4655(03)00343-6

[5] Islam, M.E., Khan, K., Akbar, M.A. and Islam, R. (2013) Enhanced (g'/g)-Expansion Method to Find the Exact Solutions of Nonlinear Evolution Equations in Mathematical Physics. Differential Equations and Applications, 1, 6-12.

[6] Cheng, X.P., Lin, J. and Wang, Z.P. (2007) Asymptotic Solutions of Perturbed NComponent Nonlinear Schrdinger Equations. Acta Physica Sinica-Chinese Edition, 56, 3031-3038.

[7] Han, L., Huang, Y. and Liu, H. (2014) Solitons in Coupled Nonlinear Schrdinger Equations with Variable Coefficients. Communications in Nonlinear Science and Numerical Simulation, 19, 3063-3073. https://doi.org/10.1016/j.cnsns.2014.01.017

[8] Biswas, A., Zhou, Q., Moshokoa, S.P., Triki, H., Belic, M. and Alqahtani, R.T. (2017) Resonant 1-Soliton Solution in Anti-Cubic Nonlinear Medium with Perturbations. Optik, 145, 14-17. https://doi.org/10.1016/j.ijleo.2017.07.036

[9] Ekici, M., Sonmezoglu, A., Zhou, Q., Moshokoa, S.P., Ullah, M.Z., Arnous, A.H., Biswas, A. and Belic, M. (2018) Analysis of Optical Solitons in Nonlinear Negative-Indexed Materials with Anti-Cubic Nonlinearity. Optical and Quantum Electronics, 50, 50-75. https://doi.org/10.1007/s11082-018-1341-3

[10] Zhong, W. and Belic, M. (2010) Traveling Wave and Soliton Solutions of Coupled Nonlinear Schrdinger Equations with Harmonic Potential and Variable Coefficients. Physical Review E, 82, Article ID: 047601. https://doi.org/10.1103/PhysRevE.82.047601

[11] Yu, F. and Yan, Z. (2014) New Rogue Waves and Dark-Bright Soliton Solutions for a Coupled Nonlinear Schrdinger Equation with Variable Coefficients. Applied Ma- 
thematics and Computation, 233, 351-358.

https://doi.org/10.1016/j.amc.2014.02.023

[12] El-Shiekh, R.M. and Gaballah, M. (2020) Solitary Wave Solutions for the Variable-Coefficient Coupled Nonlinear Schrödinger Equations and Davey-Stewartson System Using Modified Sine-Gordon Equation Method. Journal of Ocean Engineering and Science, 5, 180-185.

[13] Inc, M., Aliyu, A.I., Yusuf, A. and Baleanu, D. (2018) Optical Solitons to the Resonance Nonlinear Schrödinger Equation by Sine-Gordon Equation Method. Superlattices and Microstructures, 113, 541-549. https://doi.org/10.1016/j.spmi.2017.11.035

[14] Inc, M., Aliyu, A.I., Yusuf, A. and Baleanu, D. (2018) Optical Solitons for Biswas-Milovic Model in Nonlinear Optics by Sine-Gordon Equation Method. Optik, 157, 267-274. https://doi.org/10.1016/j.ijleo.2017.11.061 